\title{
Persistent Left Superior Vena Cava Identified during Peripherally Inserted Central Catheter Line Placement
}

\author{
Arvind Borde $^{1} \quad$ Vivek Ukirde $^{1}$ \\ 1Department of Radiology, LTMMC and GH, Sion, Mumbai, \\ Maharashtra, India
}

Int J Recent Surg Med Sci 2019;5:64-66

\begin{abstract}
Address for correspondence Arvind Borde, MD, DNB, Department of Radiology, LTMMC and GH, Sion, Mumbai 400022, Maharashtra, India (e-mail: bordeab@gmail.com).
\end{abstract}

\begin{abstract}
Keywords

- double SVC

- PICC

- PLSVC

Introduction A persistent left superior vena cava (SVC) is found in 0.3 to $0.5 \%$ of the general population. It is seen in up to $10 \%$ of the patients with a congenital cardiac anomaly, being the most common thoracic venous anomaly, and is usually asymptomatic. Being familiar with such anomaly could help clinicians avoid complications during the placement of central lines, Swan-Ganz catheters, peripherally inserted central catheter (PICC) lines, dialysis catheters, defibrillators, and pacemakers.

Case Presentation We describe a case of persistent left SVC which was noted after placement of a PICC line. A 5-year-old male child was hospitalized for evaluation and management of leukemia. He required PICC line placement for chemotherapy. He was noted to have a persistent left SVC during the procedure under fluoroscopic guidance and subsequently correct placement of PICC line in right SVC.

Discussion This anatomical variant can pose iatrogenic risks if the clinician does not recognize it. A central catheter that tracks down the left mediastinal border may also be in the descending aorta, internal thoracic vein, superior intercostal vein, pericardiophrenic vein, pleura, pericardium, or mediastinum.

Conclusion Our case is significant because the patient was diagnosed with double SVC on table only followed by the placement of PICC line into the right SVC. This case strongly demonstrates the importance of knowing the thoracic venous anomalies.
\end{abstract}

\section{Introduction}

Central venous lines (CVLs) are commonly used for therapeutic purposes for the administration of fluids, blood products, medications, nutrition, dialysis, etc. Also the CVLs can be used for diagnostic intents like central hemodynamic monitoring. Although millions of CVL placed are potentially life-saving, they are not without potentially fatal complications. Mechanical (bleeding, arterial punctures, arrhythmias, pneumothorax), thrombotic, and infectious processes are among the potential complications of a seemingly benign procedure. $^{1}$

The incidence of complications including delayed ones is estimated to be up to $33 \%$ of all placed CVLs. ${ }^{1}$ Although new technologies such as real-time ultrasound-guidance and use of dressing patches containing agents such as chlorhexidine have decreased the complications rate, CVL placements are still a significant contributor to patients' morbidity and occasionally mortality. ${ }^{2}$

The rate of CVL placement complications is typically higher in obese patients, those with a short neck, history of vasculopathy, prior CVL's placement, and less experienced clinicians. ${ }^{2}$ In the presence of venous anatomical variants, the complication rate could be much higher. ${ }^{3}$ Thus, recognizing the anatomical venous variants of the central vessels is of utmost importance to improve the safety of these procedures. One such variant is persistent left superior vena cava (PLSVC).

We present a case of PLSVC that was incidentally encountered during routine placement of a peripherally inserted central catheter (PICC) line, highlighting the radiologic findings and emphasizing the importance of familiarity to such an anomaly (-Fig. 1).
License terms

() (1) $\ominus \circledast$ 


\section{Case Presentation}

A 5-year-old male with a history of fever, fatigue, and weight loss was admitted to the pediatric intensive care unit. On routine hemogram and bone marrow aspiration he was diagnosed to have acute lymphoblastic leukemia. He was to be considered for chemotherapy (hyper-CVAD [hyperfractionated cyclophosphamide, vincristine, doxorubicin, and dexamethasone]) through PICC line. Patient was referred to interventional radiology (IR) department for guided PICC line insertion.

At IR department patient was evaluated with Doppler ultrasonography (USG) and decision was taken to insert PICC line through right cephalic vein at mid forearm under USG and fluoroscopic guidance.

While placing PICC line via right cephalic vein, PICC line initially went into the left brachiocephalic vein and coursed down in left parasternal region (-Fig. 1). It was initially suspected to have gone into left mammary vein. Subsequently, it was further advanced and PICC line curved back toward right atrial shadow crossing midline. Checking angiogram revealed coronary sinus and the diagnosis of PLSVC was made. Because coronary sinus is low flow system, in view of arrythmias, PICC line was withdrawn from left SVC and placed into the right SVC. Subsequently patient left the hospital in a stable condition.

\section{Discussion}

This case was a reminder of this common anomaly. PLSVC is the most common thoracic venous anomaly. Incidence of PLSVC is around 0.3 to $0.5 \%$ of the general population and in up to $10 \%$ of the patients with congenital cardiac anomaly. ${ }^{4}$

The cardinal veins in early embryonic development are symmetrical and bilateral; however, in later embryonic stages the left cardinal venous system normally obliterates and a new vein (future left innominate vein) drains into a right cardinal vein (future right SVC). Disturbances to this embryonic developmental stage may result in a PLSVC (-Fig. 2). ${ }^{5}$

In 70 to $90 \%$ of the patients with PLSVC, there is also a right SVC which may or may not communicate with it. ${ }^{4}$ The PLSVC commonly drains into the coronary sinus, but in up to $8 \%$ of the patients, it might drain into the left atrium. ${ }^{6}$ It is usually asymptomatic. Being familiar with such anomaly could help clinicians avoid complications during placement of central lines, Swan-Ganz catheters, PICC lines, dialysis catheters, defibrillators, and pacemakers. ${ }^{3,4}$

A central catheter that tracks down the left mediastinal border may also be in the descending aorta, internal thoracic vein, superior intercostal vein, pericardiophrenic vein, pleura, pericardium, or mediastinum. A chest X-ray along with a venous wave and a gas compatible with a venous gas may be enough to make the diagnosis of PLSVC. Other diagnostic

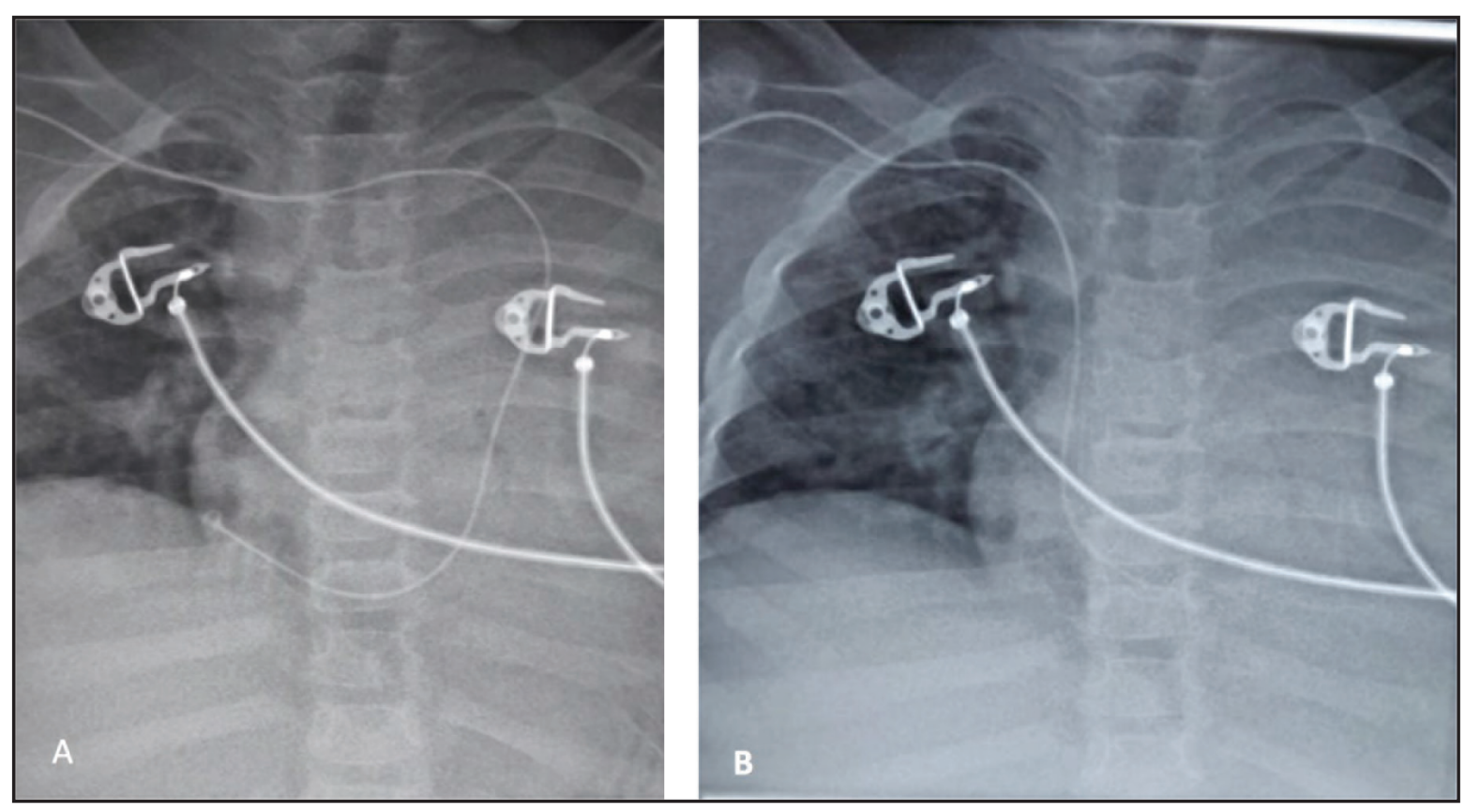

Fig. 1 (A) AP view of CXR showing a PICC line coursing down the left para-sternal region and crossing midline to right lower parasternal region-s/o of left SVC. (B) CXR of the same patient after removing the left SVC line, and placing into a right SVC. AP, anteroposterior; CXR, chest X-ray; PICC, peripherally inserted central catheter; SVC, superior vena cava. 

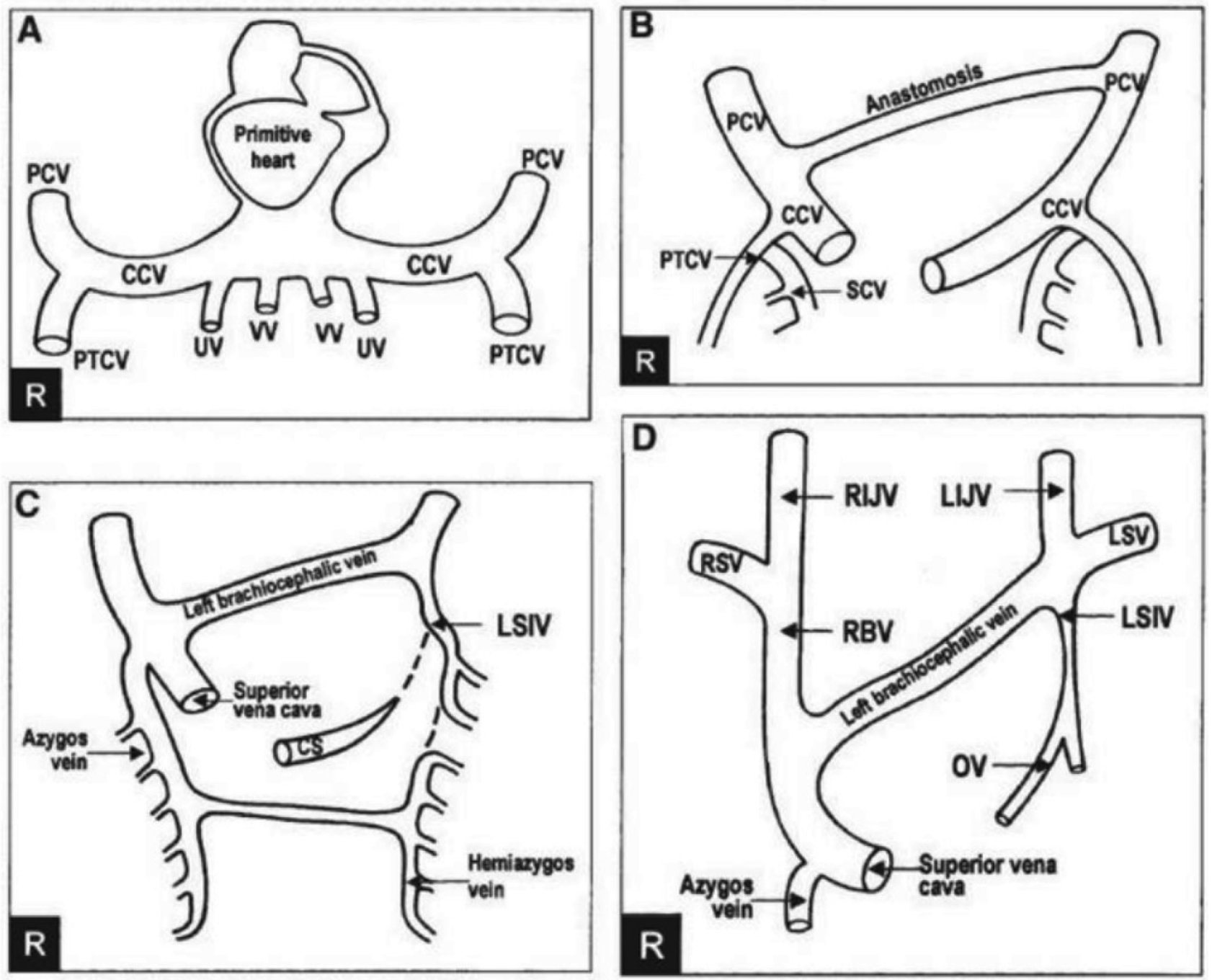

Fig. 2 Diagram illustrating normal SVC embryogenesis. (A) Initial presence of common cardinal veins. (B) Anastomosis forms creating the (C) left brachiocephalic vein. (D) Left common cardinal vein undergoes atrophy to form normal adult vasculature. CCV, common cardinal vein, CS, coronary sinus, LIJV, left internal jugular vein, LSIV, left superior intercostal vein; LSV, left subclavian vein; OV, oblique vein of the left atrium; PCV, precardinal vein; PTCV, postcardinal vein; RBV, right brachiocephalic vein; RIJV, right internal jugular vein; RSV, right subclavian vein; SCV, supracardinal vein; UV, umbilical vein; VV, vitelline vein.

modalities include computed tomography scan, magnetic resonance imaging, and echo.

\section{Conclusion}

Our case is significant because the patient was diagnosed with double SVC on table only, followed by placement into the right SVC. This case strongly demonstrates the importance of knowing the thoracic venous anomalies.

\section{Conflict of Interest}

None declared.

\section{References}

1 McGee DC, Gould MK. Preventing complications of central venous catheterization. N Engl J Med 2003;348(12):1123-1133
2 Karakitsos D, Labropoulos N, De Groot E, et al. Real-time ultrasound-guided catheterisation of the internal jugular vein: a prospective comparison with the landmark technique in critical care patients. Crit Care 2006;10(6):R162

3 Schummer W, Schummer C, Fröber R. Persistent left superior vena cava and central venous catheter position: clinical impact illustrated by four cases. Surg Radiol Anat 2003;25(3-4):315-321

4 Biffi M, Bertini M, Ziacchi M, et al. Clinical implications of left superior vena cava persistence in candidates for pacemaker or cardioverter-defibrillator implantation. Heart Vessels 2009;24(2):142-146

5 Singh B, Ramsaroop L, Maharaj J, Reddi A. Case of double superior vena cava. Clin Anat 2005;18(5):366-369

6 Ramos N, Fernández-Pineda L, Tamariz-Martel A, Villagrá F, Egurbide N, Maître MJ. Absent right superior vena cava with left superior vena cava draining to an unroofed coronary sinus [in Spanish]. Rev Esp Cardiol 2005;58(8):984-987 